\title{
Modelling and testing of optimum soil moisture levels in the corrosion of underground pipelines
}

\author{
Rukshan Azoor ${ }^{1}$, Ravin Deo ${ }^{1}$, and Jayantha Kodikara ${ }^{1, *}$ \\ ${ }^{1}$ Department of Civil Engineering, Monash University, Clayton, VIC 3800, Australia
}

\begin{abstract}
Corrosion is one of the major factors leading to the failure of buried pipelines. Soil properties such as aeration, moisture content and level of compaction are known to cause variations in the level of corrosion of buried metallic structures. It is known that, at a particular soil moisture content, the corrosion rate reaches a maximum value. While this phenomenon is generally understood, an explanation from a soil mechanics perspective with mechanisms for soil water continuity and mass transport processes is currently lacking. This work fills this void by modelling the moisture-controlled diffusion transport and electrical conductivity in soil coupled to the electrochemical activity on the buried metal surface. Variations in the electrical conductivity and oxygen diffusion in sand at different degrees of saturation were determined experimentally. The results were used as input parameters in a finite element model. Results from the coupled finite element model were compared with experimental results from electrochemical corrosion tests. The tests were conducted on cast iron specimen buried in sand and the corrosion behaviour under various aeration regimes were studied. The presence of an optimum moisture/aeration regime, where the corrosion rate becomes a maximum was demonstrated and the mechanisms behind this phenomenological behaviour are discussed in this paper. The modelling and experimental results are expected to be useful in developing non-intrusive testing methods for underground corrosion.
\end{abstract}

\section{Introduction}

Soil moisture plays an important role in underground corrosion due to its multifaceted influence on the process of corrosion [1]. In addition to facilitating the corrosion reactions by providing an aqueous medium for the dissolution of iron [2], soil moisture also controls the oxygen diffusion through soil $[3,4]$. Since underground corrosion is mass transport limited [5], the diffusion of oxygen through soil is an important factor that needs consideration. In addition, soil moisture controls the electrical conductivity of soil [6] which also has important ramifications with regard to underground corrosion [2]. Many studies on the influence of soil moisture on corrosion have been conducted, and one of the prominent findings was that there is an optimum/critical level of moisture at which the rate of corrosion is maximised [7-11]. The reasons for this behaviour were discussed in general as the competing influence of electrical conductivity and oxygen diffusion, whereby both have opposite effects on corrosion with increasing soil moisture.

While the electrical conductivity of soil is important in macro cell corrosion usually observed in the field, it has no direct impact in uniform corrosion [5], which is usually assessed in laboratory experiments. However, in uniform corrosion, the active area which is the actual area on the electrode with electrolyte contact and undergoes corrosion, is an influential factor that depends on soil moisture $[8,9,12]$. Variations of soil properties such as electrical conductivity, oxygen diffusivity and active area with soil saturation can be experimentally established. The level of corrosion in soils under different levels of moisture can also be found using standard electrochemical techniques [13]. Using these experimentally obtained relationships with soil saturation, the competing influences can be numerically modelled to establish the optimum level of moisture in a mechanistic manner. The experimental results can be then compared with the modelled curve to identify the accuracy of the mechanisms considered and to ascertain the influence of any other factors that may contribute towards the observed phenomena.

The present study is based on the aforementioned approach in order to understand the mechanisms responsible for the observed optimum soil moisture level in underground corrosion. Establishing the possible causes of the optimum soil moisture content in underground corrosion is important at both fundamental and practical level, both of which are currently lacking in this area. In this study, an attempt is made to provide the reasons for the existence of the optimum moisture level and discuss its importance in current practice of pipeline installation and condition assessment.

* Corresponding author: jayantha.kodikara@monash.edu 


\section{Experimental Methods}

\subsection{Sand characteristics}

Fine sand with an average grain size of 150 microns was used in this study. Known amount of sand was mixed with appropriate amount of $0.001 \mathrm{M} \mathrm{NaCl}$ solution to prepare a series of sand samples with the required moisture levels. These samples compacted to approximately the same dry density were used for the oxygen diffusion, electrical conductivity, and electrochemical polarisation experiments as discussed next.

\subsection{Oxygen diffusion in soil}

The oxygen diffusion coefficient of the samples were determined using the diffusion chamber method [14]. The modified UMS-G apparatus developed by Rouf et al [15] shown in Fig 1 was used for this purpose. The sample holder was modified with a 3D printed mesh. The sand samples were compacted into the sample holder, one at a time and measurements were conducted. For the compacted sample, the dry density was noted as this level of compaction was also utilised in the other experiments. Measurements were conducted by initially loading the sample holder into the apparatus and purging the bottom chamber of oxygen. Oxygen was then allowed to diffuse from the atmosphere, through the sample, into the bottom chamber. The oxygen concentration was measured and recorded at 1-minute intervals using a data acquisition system. The measured concentrations were converted to relative concentrations and Fick's second law was used to compute the oxygen diffusion coefficient. The solution for the relative concentration developed by Carslaw and Jaeger[16] was used for this purpose.

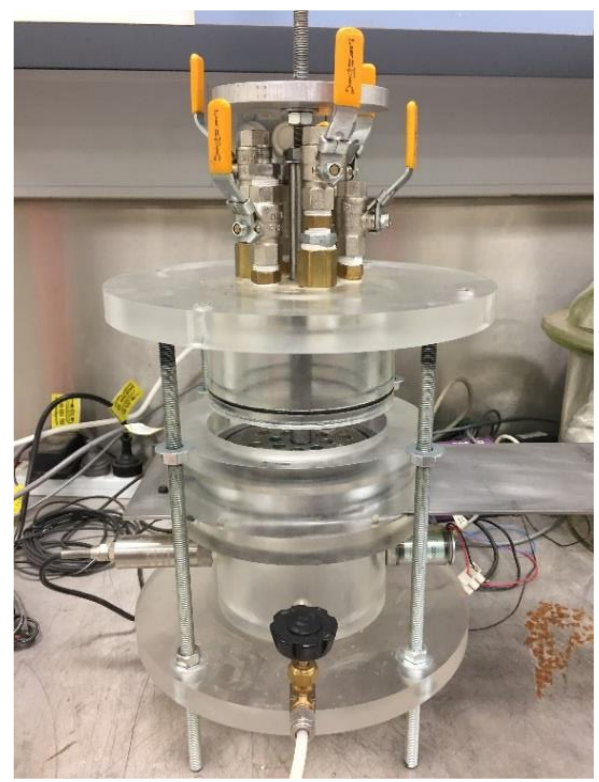

Fig. 1. The UMS-G experimental apparatus used for oxygen diffusion tests

\subsection{Soil electrical conductivity}

The electrical conductivity of the sand samples at similar dry densities, as in the oxygen diffusion tests, were measured using a 4-electrode setup described in [2] and shown in Fig. 2. This setup conforms to the design criteria of [17] and applies a current pulse between two end-cap copper electrodes and measures the potentials between two middle non-polarisable electrodes. The current/potential response was transformed to the soil conductivity (reciprocal of resistivity) using the geometrical factor of the sample holder. Prior to measurements of sand samples, the experimental setup was calibrated with solutions of known conductivity for quality control purposes.

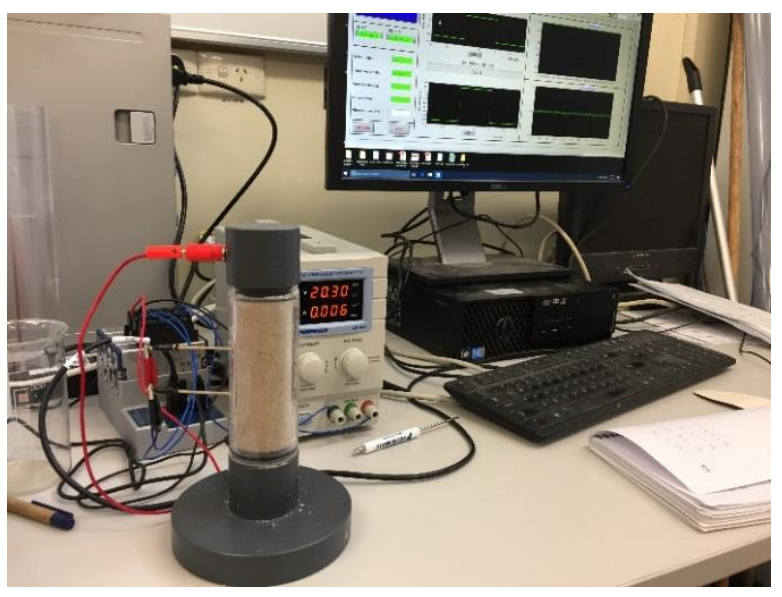

Fig. 2. Soil resistivity measurement setup.

\subsection{Polarisation experiments}

The corrosion rates and corrosion potentials were obtained using potentiodynamic polarisation experiments. Due to the relatively high resistivity of soils, conventional electrochemical cells with standard electrode placements cannot be used to conduct the electrochemical tests. Therefore, a special cell assembly allowing the placement of electrodes to minimise resistive effects was designed and $3 \mathrm{D}$ printed for this purpose. Fig. 3 shows the 3D printed cell assembly and the electrode arrangement. The working electrode with the corroding metal was placed at the bottom of the cell ensuring a flat surface to allow soil compaction. The miniature $\mathrm{Ag} / \mathrm{AgCl}$ reference electrode was inserted through the centre of the cylinder and its tip rested about $1 \mathrm{~mm}$ above the working electrode. The counter electrode was inserted into the soil from the side as shown in Fig.3. 


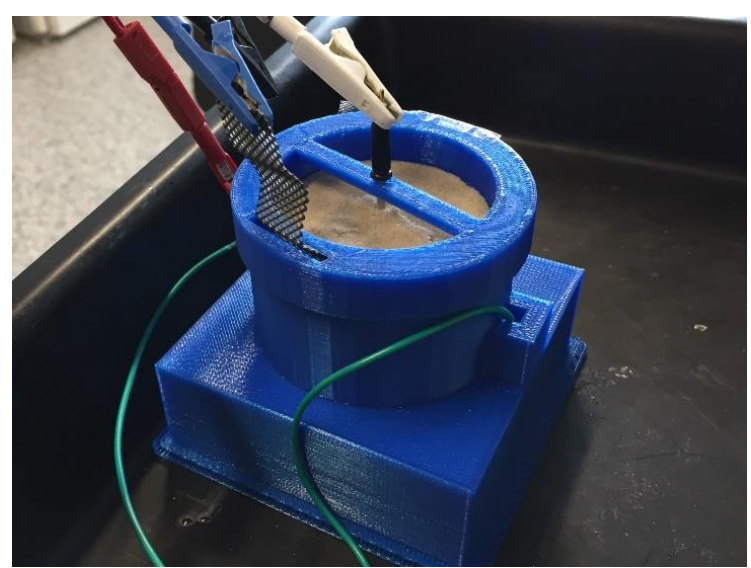

Fig. 3. 3D printed electrochemical cell assembly with electrodes for soil polarisation test

The sand samples were compacted into the electrochemical cell, on top of the cast iron working electrode to different degrees of saturation while maintaining approximately the same dry densities as in the oxygen diffusion and electrical conductivity experiments. The reference and counter electrodes were carefully inserted into the compacted sand and was connected to the BioLogic(C) potentiostat. Prior to polarisation tests, the open circuit potential was monitored for a period of 30 minutes to ensure equilibrium conditions. The corrosion rates and corrosion potentials from the experimental polarisation curves were estimated through Tafel curve fits [18].

\subsection{Active Area Measurement}

The area on the working electrode that actually undergoes corrosion is known as active area $[8,9]$. This area corresponds to the electrolyte contact area in the soil-metal interface. To ascertain the active area, the corroded electrode surfaces were digitally imaged after each polarisation test. Subsequently, the images were thresholded using Otsu's algorithm [19] and the active area, normalised to the total electrode area, were determined [12]. The relationship between the active area and the degree of saturation was established in this manner. Since the active area influences the corrosion current densities [8,9], it was considered in the numerical model equations.

\section{Numerical Methods}

A steady state numerical model was developed to simulate the effects of varying degree of saturation on the corrosion rate. The experimental results from the oxygen diffusivity and electrical conductivity tests were used as input functions to characterise the soil medium as an electrolyte that facilitates corrosion. A 1D model was developed to scrutinise influencing factors pertaining to the optimum moisture content in corrosion, and a 3D model was developed for illustration of a possible practical application.

\subsection{Corrosion electrochemistry}

Assuming that the anodic and cathodic reactions are given by Eqs. 1 and 2,

$$
\begin{gathered}
\mathrm{Fe} \rightarrow \mathrm{Fe}^{2+}+2 \mathrm{e}^{-} \\
\mathrm{O}_{2}+2 \mathrm{H}_{2} \mathrm{O}+4 \mathrm{e}^{-} \rightarrow 4 \mathrm{OH}^{-}
\end{gathered}
$$

The following equations were used to define the anodic and cathodic current densities,

$$
\begin{gathered}
i_{F e}=\frac{1}{1+\exp \left(10 \times\left(0.4-S_{r}\right)\right)} i_{F e}^{0} \times 10^{\frac{\eta_{F e}}{A_{F e}}} \\
i_{O_{2}}=\frac{C_{O_{2}}}{C_{O_{2}}^{r e f}} \times i_{O_{2}}^{0} \times 10^{\frac{\eta_{O_{2}}}{A_{O_{2}}}}
\end{gathered}
$$

where, $S_{r}$ is the degree of saturation, $i_{F e}\left(\mathrm{~A} / \mathrm{m}^{2}\right)$ and $i_{O_{2}}\left(\mathrm{~A} / \mathrm{m}^{2}\right)$ are the anodic and cathodic current densities respectively, and $i_{F e}^{0}\left(\mathrm{~A} / \mathrm{m}^{2}\right)$ is the exchange current density for the anodic reaction, $i_{O_{2}}^{0}\left(\mathrm{~A} / \mathrm{m}^{2}\right)$ is the exchange current density for the cathodic reaction, $A_{F e}(\mathrm{~V})$ and $A_{O_{2}}$ (V) are the anodic and cathodic Tafel slopes respectively, $C_{\mathrm{O}_{2}}\left(\mathrm{~mol} / \mathrm{m}^{3}\right)$ is the oxygen concentration in the soil electrolyte and $C_{O_{2}}^{r e f}\left(\mathrm{~mol} / \mathrm{m}^{3}\right)$ is the atmospheric reference oxygen concentration. $\eta_{F e}(\mathrm{~V})$ is the anodic over-potential, and $\eta_{\mathrm{O}_{2}}(\mathrm{~V})$ is the cathodic overpotential, which are given by Eqs. 5 and 6 respectively:

$$
\begin{aligned}
& \eta_{\mathrm{Fe}}=-\phi-E_{\mathrm{Fe}}^{e q} \\
& \eta_{\mathrm{O}_{2}}=-\phi-E_{\mathrm{O}_{2}}^{e q}
\end{aligned}
$$

where $\phi(\mathrm{V})$ is the electrolyte potential. The anodic current density (Eq. 3) includes the active area concept $[8,9]$, while the cathodic current density expression includes the concentration polarisation effect [20]. In this manner both the anodic and cathodic reactions cater to the practical observations in underground corrosion.

The electrolyte potential $(\phi)$ in the soil electrolyte is governed by Eq. 7 ,

$$
\nabla \cdot(\sigma \nabla \phi)=0
$$

where, $\sigma(\mathrm{S} / \mathrm{m})$ is the soil electrical conductivity. The current in the electrolyte, $\mathbf{i}_{l}\left(\mathrm{~A} / \mathrm{m}^{2}\right)$, is governed by Ohm's law (Eq. 8) and is related to the anodic and cathodic current densities as shown in Eq. 9.

$$
\begin{aligned}
& \mathbf{i}_{l}=-\sigma \nabla \phi \\
& \mathbf{n . i _ { l }}=i_{F e}-i_{O 2}
\end{aligned}
$$

\subsection{Oxygen diffusion}

The steady state governing equation for the oxygen concentration is given by Eq. 10 .

$$
\nabla \cdot\left(D_{e} \nabla C_{\mathrm{O}_{2}}\right)=0
$$

where, $D_{e}\left(\mathrm{~m}^{2} / \mathrm{s}\right)$ is the effective diffusion coefficient for oxygen and $\mathrm{C}_{\mathrm{O}_{2}}$ is the oxygen concentration in the soil. 
$D_{e}$ is input as a function obtained from the aforementioned experimental results. A constant concentration boundary condition is imposed at the surface exposed to the atmosphere and the oxygen diffusing to the corroding metal surface is assumed to be consumed by the cathodic reaction as shown by Eq. 11 .

$$
i_{O_{2}}=-D_{e}\left(\mathbf{n} . \nabla C_{O_{2}}\right) z F
$$

where, $z$ is the number of electrons involved in the reaction and $F$ is the Faraday constant. The oxygen flux coupled with the cathodic corrosion current density together captures the diffusion limitation conditions in underground corrosion.

\section{Results and Discussion}

\subsection{Experimental and model results}

Apart from the corrosion rates and corrosion potentials, the anodic $\left(\beta_{a}\right)$ and cathodic $\left(\beta_{c}\right)$ Tafel slopes were also determined from the polarisation experiments. While the slopes show some variability with the $S_{r}$, average values of $140 \mathrm{mV}$ and $120 \mathrm{mV}$ were determined for the anodic and cathodic slopes respectively. These average values were used in the corrosion kinetics equations in the numerical model.

The results from the oxygen diffusion and soil electrical conductivity tests are summarized in Fig. 4. It is observed that the oxygen diffusion coefficient decreases with increase in the degree of saturation, while the electrical conductivity increases with increase in the degree of saturation. These two properties as functions of the degree of saturation were used as inputs in the numerical model. It should be noted that for uniform corrosion, with no macro cell activity, there is no influence from the electrical conductivity. However, in the presence of a net current through the electrolyte, indication of macro cell corrosion, the electrical conductivity becomes important.

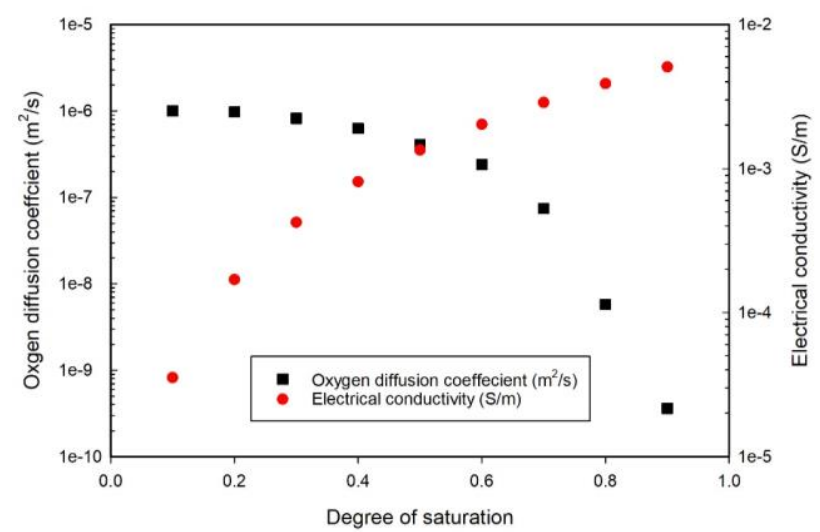

Fig. 4. Oxygen diffusion coefficient and electrical conductivity calculated form the experiments for different degrees of saturation in sand

The relationship between active area and the degree of saturation was found to be best described by a sigmoidal function. The sigmoidal shape of the active area is also backed by some studies on soil water distribution and electrical properties soil [10,21]. Fig 5 shows the experimental results from the active area and the fitted sigmoidal curve.

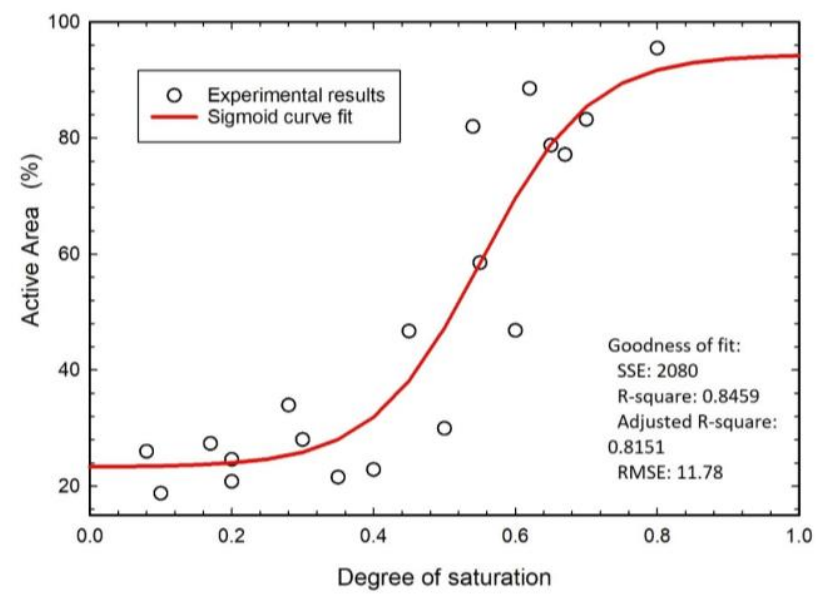

Fig. 5. The relationship between active area and the degree of saturation showing an approximately sigmoid shape

Fig. 6 shows the corrosion current density (i-corr) $\left(\mathrm{A} / \mathrm{m}^{2}\right)$ values computed form the numerical model overlaid on the experimental results of i-corr. In the case of uniform corrosion simulated here, the increase in corrosion current density with soil water saturation is attributed to the effect of active area [9], since the electrolyte contact on the metal surface increases with increasing moisture levels. The subsequent decrease in the current density after reaching the peak point, is due to the restriction of oxygen diffusion to the metal surface brought about by the filling up of the air pore space with water, making the air phase discontinuous [22]. As diffusion of oxygen through the water phase is several orders of magnitude smaller than in the air phase, the corrosion rate starts to decrease with insufficient oxygen at higher saturations. These two competing factors are modelled giving rise to the characteristic shape of the curve and the optimum point. While the model results show similarity with the experimental, a notable difference in the peak point of the corrosion rates is noted. The experimental values show a clear peak at around $S_{r}=0.5$, while the numerical model results attain the peak much later $\left(S_{r} \approx 0.8\right)$. A possible reason for this behaviour is that at relatively high saturations, water settles down at the bottom of the cell due to gravity, thereby increasing the degree of saturation close to the working electrode compared to the rest of the cell. This means that the oxygen deficiency would take place much earlier leading to a decrease in corrosion rate at relatively lower overall saturations. Gravity drainage effects and soil suction-based water movement is not modelled in the present model; hence these effects are not discussed in detail. The effect of soil suction in the optimum moisture content in reporting optimum moisture content in soil is considered in a separate study. 


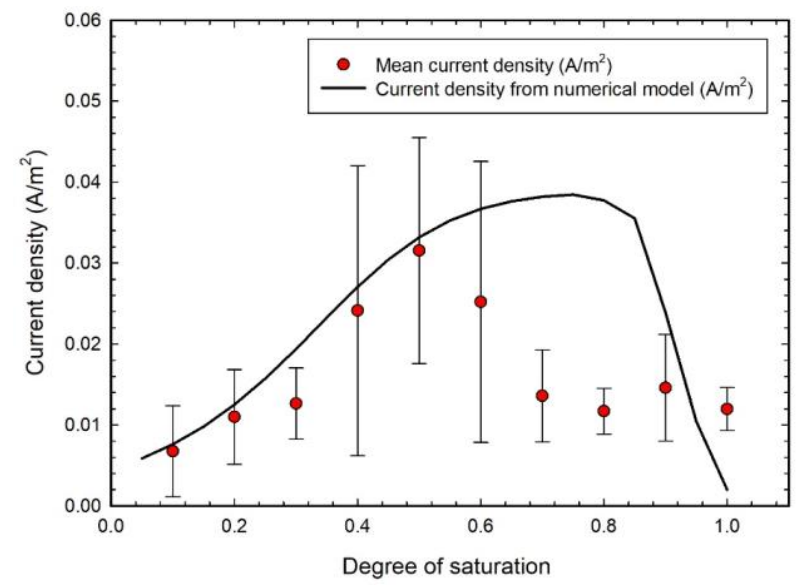

Fig. 6. The experimental current densities and the current densities from the model simulation for different saturation levels in sand

Fig 7 shows the results of a parametric sweep of the soil electrolyte length in the 1D model. This length corresponds to the diffusion length through soil and therefore simulates the burial depth of underground pipelines. It is seen that both the maximum corrosion rate and the degree of saturation at the optimum point decreases with increasing depth. This result can be explained by the diffusion control in soil corrosion. Since one of the competing factors controlling the optimum point is oxygen diffusion, any factor that influences the process of diffusion will in turn influence the rate of corrosion. This means that the burial depth of the corroding metal is an important factor that needs to be reported, when dealing with the optimum moisture content. For the sand considered in the present study, it is seen that at a typical burial depth of $1 \mathrm{~m}$ the optimum corrosion occurs at around $S_{r}=0.6$. It is also noted that the decrease in corrosion rate with increasing depth has been observed in practice and has been reported in early works by Tomashov [5]. However, to the authors' knowledge, the dependency of the optimum moisture level with the burial depth has not been reported before.

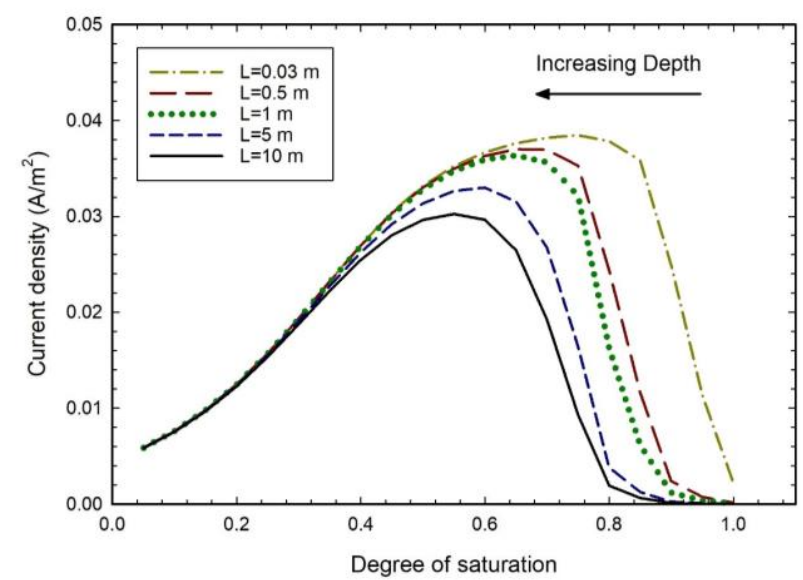

Fig. 7. Results from a parametric sweep changing the diffusion length (L) Variations in the current density with the diffusion length, $L$.

\subsection{Practical implications}

A 3D numerical simulation was conducted to assess the practical use of the results reported in this work. A hypothetical situation of a homogeneous sand subsurface variably saturated in the range $0 \leq S_{r} \leq 1$ (linearly in the $\mathrm{x}$ direction, as shown on the top surface) was simulated with a constant concentration boundary condition imposed on the top surface ( $\mathrm{z}=0$ plane). A ferrous pipe traversing through this sand medium and the corrosion rate on the pipe surface was assessed. Note, in the present work the gravity effects on moisture distribution and the variations in $S_{r}$ with depth were not considered. Fig 8 shows the corrosion current density on the pipe surface and the net current density in the soil electrolyte.

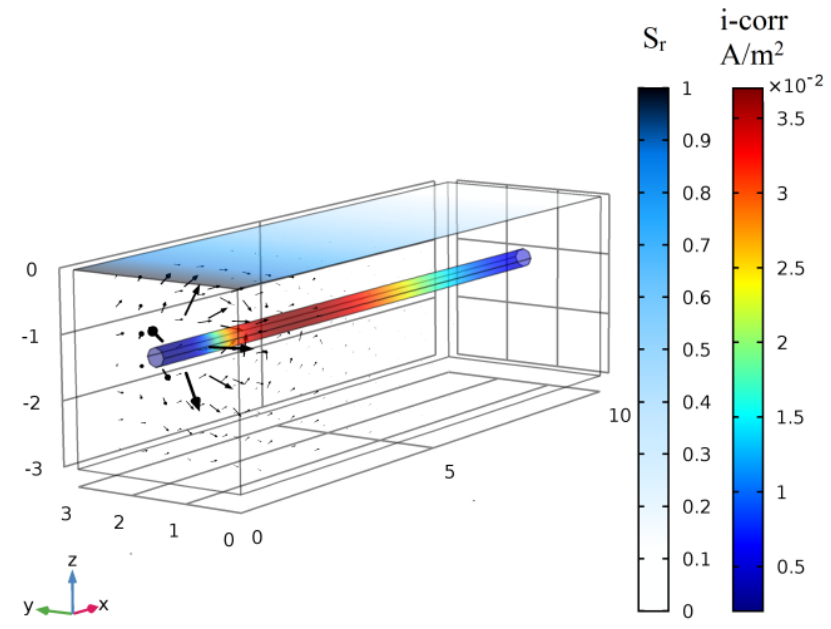

Fig. 8. Corrosion current density on the pipe surface and the electrolytic current flowing through the soil denoted by arrows. The $S_{r}$ distribution is shown on the top surface ( $\mathrm{z}=0$ plane). Note that gravity drainage effects are not considered and the same surface $S_{r}$ distribution is maintained with depth.

The maximum corrosion rate was observed at the location of the pipe corresponding to the optimum degree of saturation reported earlier. It is also seen from the electrolyte current that macro cell currents established due to the effects of differential aeration (variably saturated). The highly saturated area turned anodic with a net current leaving the pipe surface. These macro cells can evolve into intensely localised corrosion through the spatial separation of anodes and cathodes. The soil electrical conductivity plays a significant role in macro cell corrosion. However, this study does not consider factors that accentuate such effects such as passivation and corrosion product deposition. These effects and their time-dependent influence on corrosion were discussed in a previous paper [12]. The 3D simulation involving a hypothetical saturation distribution shows that the level of corrosion becomes a maximum corresponding to the optimum point, while also highlighting the development of macro cells due to 
differential aeration. This means that even waterlogged areas need to be considered as high priority areas in corrosion assessment due to the possibility of coupled macro cell corrosion. The subsurface moisture levels in soil can be ascertained by several geophysical methods such as electrical resistivity tomography and ground penetrating radar (GPR) $[23,24]$ and their applications have been previously discussed. GPR can also be used for remotely assessing possible conditions of corroded pipes [25], and can be used in conjunction with other assessment methods for testing corrosivity of soil in field conditions.

\section{Conclusions}

The present study investigated the optimum/critical moisture content at which corrosion rate in soils reaches a maximum, using numerical models supported with experiments. It was demonstrated that the active area of corrosion and oxygen diffusion can partly explain the occurrence of the optimum moisture level, where the corrosion rate attains a maximum value. It is noted that additional factors that govern the moisture distribution in soil such as soil suction, and other soil dependent properties, will need to be incorporated to improve the accuracy of the modelling efforts. These features will be addressed in future studies. The importance of soil depth in the optimum soil moisture conditions was also demonstrated. This observation needs a careful examination on the current practice of buried pipe installation. Moreover, the practical relevance of the work was demonstrated. It was shown that assessment of subsurface moisture levels using geophysical techniques can be used in soil corrosivity and pipe condition assessments.

\section{References}

1 I. S. Cole, D. Marney Corros. Sci. 56 5-16 (2012)

2 R. N. Deo, J. P. Cull Geotech. Test. J. 38 965-77 (2015)

3 M. Aachib, M. Mbonimpa, M. Aubertin Water. Air. Soil Pollut. 156 163-93 (2004)

4 E. Yanful J. Geotech. Eng. 119 1207-28 (1993)

5 N. D. Tomashov Theory of Corrosion and Protection of Metals (Macmillan, 1966) pp 399421 (1966)

6 Y. Mualem, S. P. Friedman Water Resour. Res. 27 2771-7 (1991)

7 S. K. Gupta, B. K. Gupta Corros. Sci. 19 171-8 (1979)

8 D. N. Dang, L. Lanarde, M. Jeannin, R. Sabot, P. Refait Electrochim. Acta 176 1410-9 (2015)

9 R. Akkouche, C. Rémazeilles, M. Jeannin, M. Barbalat, R. Sabot, P. Refait Electrochim. Acta 213 698-708 (2016)

10 J. Jiang, J. Wang, W. Wang, W. Zhang Electrochim. Acta 54 3623-9 (2009)

11 R. N. Deo, N. Birbilis, J. P. Cull Corros. Sci. 80 339-49 (2014)
12 R. M. Azoor, R. N. Deo, N. Birbilis, J. K. Kodikara Corrosion 74 1177-91 (2018)

13 W. S. Tait An Introduction to Electrochemical Corrosion Testing for Practicing Engineers and Scientists (Wisconsin: PairODocs Publications) (1994)

14 J. a Currie Br. J. Appl. Phys. 11 314-7 (1960)

15 M. A. Rouf, A. Bouazza, R. M. Singh, W. P. Gates, R. K. Rowe Can. Geotech. J. 53 1000-12 (2016)

16 H. S. Carslaw, J. C. Jaeger Conduction of Heat in Solids (1959)

17 E. Zimmermann, A. Kemna, J. Berwix, W. Glaas, H. Vereecken Meas. Sci. Technol. 19 (2008)

18 R. Baboian Corrosion tests and standards: application and interpretation (ASTM international) (2005)

19 N. Otsu IEEE Trans. Syst. Man. Cybern. 9 62-6 (1979)

20 S. C. Kranc, A. A. Sagues Corrosion 50 50-61 (1994)

21 T. Saarenketo J. Appl. Geophys. 40 73-88 (1998)

22 J. Kodikara Can. Geotech. J. 49 1227-43 (2012)

23 R. Deo Geophysical methods for assessments of soil corrosivity (Monash University) (2013)

24 R. N. Deo, R. M. Azoor, C. Zhang, J. K. Kodikara J. Appl. Geophys. (2017)

25 R. N. Deo, R. M. Azoor, J. K. Kodikara Advanced Ground Penetrating Radar (IWAGPR), 2017 9th International Workshop on (Edinburgh, United Kingdom, United Kingdom) pp 1-5 (2017) 